\title{
Short-range structural and magnetic order in rapidly-solidified Ag-Mn alloys
}

\author{
F. Jiménez-Villacorta, ${ }^{1,2}$ I. Puente-Orench,,${ }^{3,4}$ J. Rodríguez-Carvajal ${ }^{4}$ and L. H. Lewis ${ }^{1}$ \\ ${ }^{I}$ Department of Chemical Engineering, Northeastern University, Boston MA 02115, USA \\ ${ }^{3}$ Instituto de Ciencia de Materiales de Madrid, CSIC, Cantoblanco, 28049 Madrid, Spain \\ ${ }^{3}$ Institut Laue-Langevin, 6 rue Jules Horowitz, 38042 Grenoble, France \\ ${ }^{3}$ Instituto de Ciencia de Materiales de Aragón, CSIC-Universidad de Zaragoza, 50009 Zaragoza, Spain
}

\section{ABSTRACT}

Short-range structural and magnetic order in rapidly solidified nanostructured $\mathrm{Ag}_{100-\mathrm{x}} \mathrm{Mn}_{\mathrm{x}}$ alloys featuring nominal large Mn content $(x=25,32,40$ at.\%), well above the maximum equilibrium concentration ( $x \sim 14$ at.\%), are quantified using x-ray, neutron and magnetic probes. These inhomogenous alloys contain coexisting Mn-rich and Mn-deficient nanoscopic face-centeredcubic-type phases. Interaction between these regions provides large exchange bias at low temperature, with the magnitude of the unidirectional anisotropy field increasing gradually with increased $\mathrm{Mn}$ concentration. Neutron diffraction data reveal no evidence of long-range antiferromagnetic ordering but instead identifies short-range structural order from the presence of a diffuse scattering peak that evolves with Mn content. Moderate annealing temperatures $\left(T_{a n n}=350\right.$ ${ }^{\circ} \mathrm{C}$ ) promote homogenization of the Mn content in the alloys with subsequent suppression of exchange bias, confirming the above model. A strong interfacial exchange density between Mndeficient and the Mn-rich nanocrystalline phases exists that is determined to be of the same order of magnitude as that found in other exchange-biased ferromagnetic/antiferromagnetic systems. The intriguing behavior of these AgMn alloys as a function of temperature, of Mn content and of annealing condition highlights options to manipulate and tailor their magnetic attributes such as interface exchange and magnetic anisotropy. 


\section{INTRODUCTION}

The exchange bias effect that arises from intimate exchange coupling between a ferromagnetic (FM) and an antiferromagnetic (AF) phase ${ }^{1,2}$ is a key enabler for the development of spintronic devices ${ }^{3,4}$ and may hold promise for application in permanent magnet technology as socalled "exchange-bias" magnets. 5,6 Properly engineered, exchange bias donates enhancements to unidirectional magnetic anisotropy that may stabilize the magnetization ${ }^{7}$ to yield improved remanence, ${ }^{8}$ coercivity ${ }^{9}$ and maximum energy product, $(B H)_{\max .}{ }^{1,10,11}$ Among metallic AF materials, manganese-based binary systems such as $\mathrm{Fe}-\mathrm{Mn},{ }^{12} \mathrm{Ir}_{-} \mathrm{Mn}^{13}$ and $\mathrm{Cu}-\mathrm{Mn}^{14}$ hold potential as components in exchange-biased magnets because their magnetic properties may be tailored by compositional variation. ${ }^{15,16}$ However, analogous to the case of exchange-spring nanocomposites, ${ }^{17}$ incorporation of the anisotropy-donor phase (which is the high-coercivity phase in exchange-spring magnets or the antiferromagnetic phase in exchange-bias magnets) is accompanied by a decrease of the overall magnetization, requiring optimization of the nanocomposite phase volume ratio. ${ }^{18,19}$ In this current work, the structural and magnetic properties of $\mathrm{Ag}_{100-\mathrm{x}} \mathrm{Mn}_{\mathrm{x}}$ alloys with $\mathrm{Mn}$ concentrations $(25 \leq x \leq 40$ at.\%) retained beyond the equilibrium content are reported. In contrast to equilibrium compositions in the Ag-Mn binary phase diagram with a maximum $\mathrm{Mn}$ content of $\sim$ 14 at. $\%^{20}$, rapid solidification allows metastable retention of a large fraction of $\mathrm{Mn}$ in the Ag-Mn alloys. Solidification of melted alloys by melt spinning is used provides very high cooling rates of up to $10^{6} \mathrm{~K} / \mathrm{s}^{21}$ The quenched Ag-Mn alloys fosters phase separation, chemical disproportionation and a resultant large exchange bias at low temperatures. ${ }^{22}$ The origin of such unidirectional anisotropy, recently observed as well in MnAl and Mn-based Heusler melt-spun alloys, ${ }^{23,24,25}$ resides in fluctuations of $\mathrm{Mn}$ content that produces adjacent exchange-coupled nanoscopic regions with predominant FM and AF character. 
The Ag-Mn alloys share a rich history with the canonical Au-Mn and Cu-Mn systems ${ }^{26,27,28}$ that exhibit spin glass behavior at low temperatures ${ }^{29,30}$. Recently, modified transport and magnetic properties of nanocrystalline AgMn relative to that characterizing it larger-grained counterpart have been observed in alloys of low Mn content $\mathrm{t}^{30,31,32}$. The seminal work of Morris and Williams ${ }^{33}$ on the magnetic susceptibility of AgMn alloys containing Mn up to 38.1 at.\%, does not mention exchange anisotropy effects in this system. Kouvel studied AgMn alloys with a moderately enriched Mn content (in the range 11.9 to 23.7 at.\% Mn) and identified both spin-glass-like features and exchange bias in these alloys ${ }^{34,35}$ that were attributed to the formation FM and AF regions arising from random statistical fluctuations of the Mn content. In every case it has been reported that an increased Mn concentration conveys an increased blocking temperature $T_{B}$, defined as the transition temperature between the magnetic spin-glass state and the paramagnetic state, signaled by a cusp in the magnetization $v s$. temperature $(M v s . T)$ curves.

This current work reports on magnetic properties and structure of nanocrystalline Ag100${ }_{\mathrm{x}} \mathrm{Mn}_{\mathrm{x}}$ alloys with a high metastable Mn content, $x=25,32,40$ at.\%, prepared by melt spinning. Rapid solidification techniques and subsequent quenching yields an increased solid solubility and grain refinement. ${ }^{36}$ The interatomic exchange coupling and exchange anisotropy of these alloys are determined to vary systematically with variation in the Mn content. It is envisioned that increased understanding of the tunability of the magnetic features of Mn-based alloys at low temperatures will donate new ideas for increased stability of magnetic media, permanent magnet and exchange-biased magnetic sensor technologies. ${ }^{37,38}$

\section{EXPERIMENTAL}

Silver-manganese $\left(\operatorname{Ag}_{100-\mathrm{x}} \mathrm{Mn}_{\mathrm{x}}, x=25,32,40\right.$ at.\%) ribbons were prepared by melt-spinning in an argon atmosphere from pre-alloyed AgMn (99.98\% purity) arc-melted charges. During the 
melt-spinning process, the nozzle-to-wheel distance was fixed at $3 \mathrm{~mm}$ and a tangential wheel speed of $31 \mathrm{~m} / \mathrm{s}$ was employed during the quench. The resultant ductile ribbons possessed thicknesses 50-100 $\mu \mathrm{m}$ and widths of 3-4 mm. The morphology and compositional profile of the quenched ribbons, as well as those of the post-alloyed charges, were evaluated with scanning electron microscopy (SEM) and in-situ energy-dispersive x-ray spectroscopy (EDX; Hitachi S4800). The sample crystallinity and phase constitution were examined by x-ray diffraction (XRD) using $\mathrm{Cu}-\mathrm{K}_{\alpha}$ radiation (Philips XPert Pro); lattice parameters were determined using a least-squares cell-parameter fitting program ${ }^{39}$. The average crystallographic grain dimension $D$ and the lattice distortion ratio $\eta$, which provides an estimation of the microstrain effects in the lattice, were determined using the Williamson-Hall relationship ${ }^{40,41}$ applied to the XRD data:

$$
\beta_{\text {total }}=\beta_{\text {size }}+\beta_{\text {strain }}=\frac{k \lambda}{D \cos \theta}+4 \eta \frac{\sin \theta}{\cos \theta}
$$

where $\beta_{\text {total }}$ is the full-width at half-maximum of the Bragg diffraction peak, $\eta=\Delta d / d$ is the strain parameter, $\Delta d$ is the lattice $d$ spacing deviation, $k$ is the Scherrer constant $(k \approx 0.9), \lambda$ is the incident wavelength, and $\theta$ is the diffraction angle.

Neutron diffraction experiments were performed in the high-flux two-axis D1B diffractometer at the Institut Laue-Langevin (ILL). Diffraction patterns were collected at selected temperatures from 2 to $300 \mathrm{~K}$ using a calibrated wavelength of $\lambda=1.2899 \AA$. The $\operatorname{Ag}_{100-\mathrm{x}} \mathrm{Mn}_{\mathrm{x}}$ ribbons where cut into very small pieces for measurement and were sealed into a 6-mm cylindrical vanadium container that was placed inside a cryostat to control the measurement temperature. The crystal structure was refined by applying the Rietveld method to the data using the FullProf Suite ${ }^{42}$.

Magnetic characterization was carried out using a Quantum Design MPMS-5 Superconducting Quantum Interference Device (SQUID) magnetometer. Zero-field cooled - field- 
cooled (ZFC-FC) magnetothermal curves were measured from in the temperature range $10 \mathrm{~K} \leq T \leq$ $350 \mathrm{~K}$ under an applied field $H_{a p p l}$ of $1 \mathrm{kOe}$. Field-cooled $\left(H_{a p p l}=50 \mathrm{kOe}\right)$ and zero-field cooled $M(H)$ loops have been collected at several temperatures in the range $10 \mathrm{~K}-300 \mathrm{~K}$. The intrinsic coercive field $H_{C i}$, defined as the reversal field in the descending branch, the exchange bias field $H_{E}$, defined as the average midpoint between the reversal fields of both ascending and descending branches, and the unidirectional anisotropy coefficients were obtained after subtracting the linear contribution of the $M(H)$ loops, following the method of Kouvel ${ }^{34}$. Such experimental linear contribution originates from the linear susceptibility of the antiferromagnetic (cluster-glass) phase (present below the blocking temperature) and the paramagnetic phase (present above the blocking temperature) of the alloys.

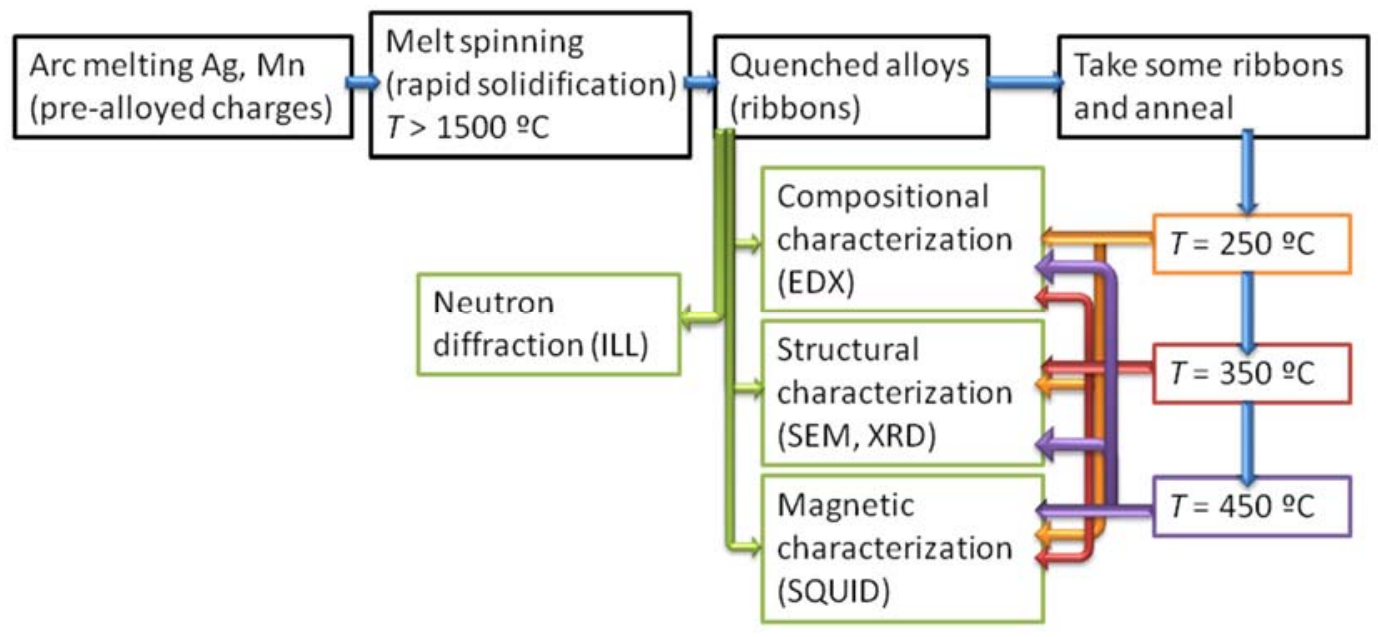

Fig. 1. Experimental diagram describing the experimental procedure (fabrication and characterization steps).

Finally, correlations between the magnetic response and the microstructural evolution were investigated in the sample with the composition $\mathrm{Ag}_{68} \mathrm{Mn}_{32}$ by subjecting it to successive isochronal annealing treatments for 30 minutes in the temperature range $250-450{ }^{\circ} \mathrm{C}$ while vacuum sealed $(\sim$ $10^{-6}$ Torr) in a $2.5-\mathrm{mm}$ diameter quartz tube to prevent oxidation ${ }^{43}$. The microstructure as well as the magnetic response of the annealed $\mathrm{Ag}_{68} \mathrm{Mn} 32$ sample was routinely examined after every 
annealing treatment. An experimental process diagram describing the experimental procedure explained above is depicted on Figure 1.

\section{RESULTS}

The as-quenched Ag-Mn alloys are confirmed to be homogeneous on the microscale in their as-solidified state (see SEM micrographs on Fig. 2), as rapid solidification may reduce intergrain microsegregation inducing simultaneous extended solid solubility. ${ }^{44,45}$ Nevertheless, an increasing roughness and density of small droplets are apparent at the surface with increasing $\mathrm{Mn}$ concentration.
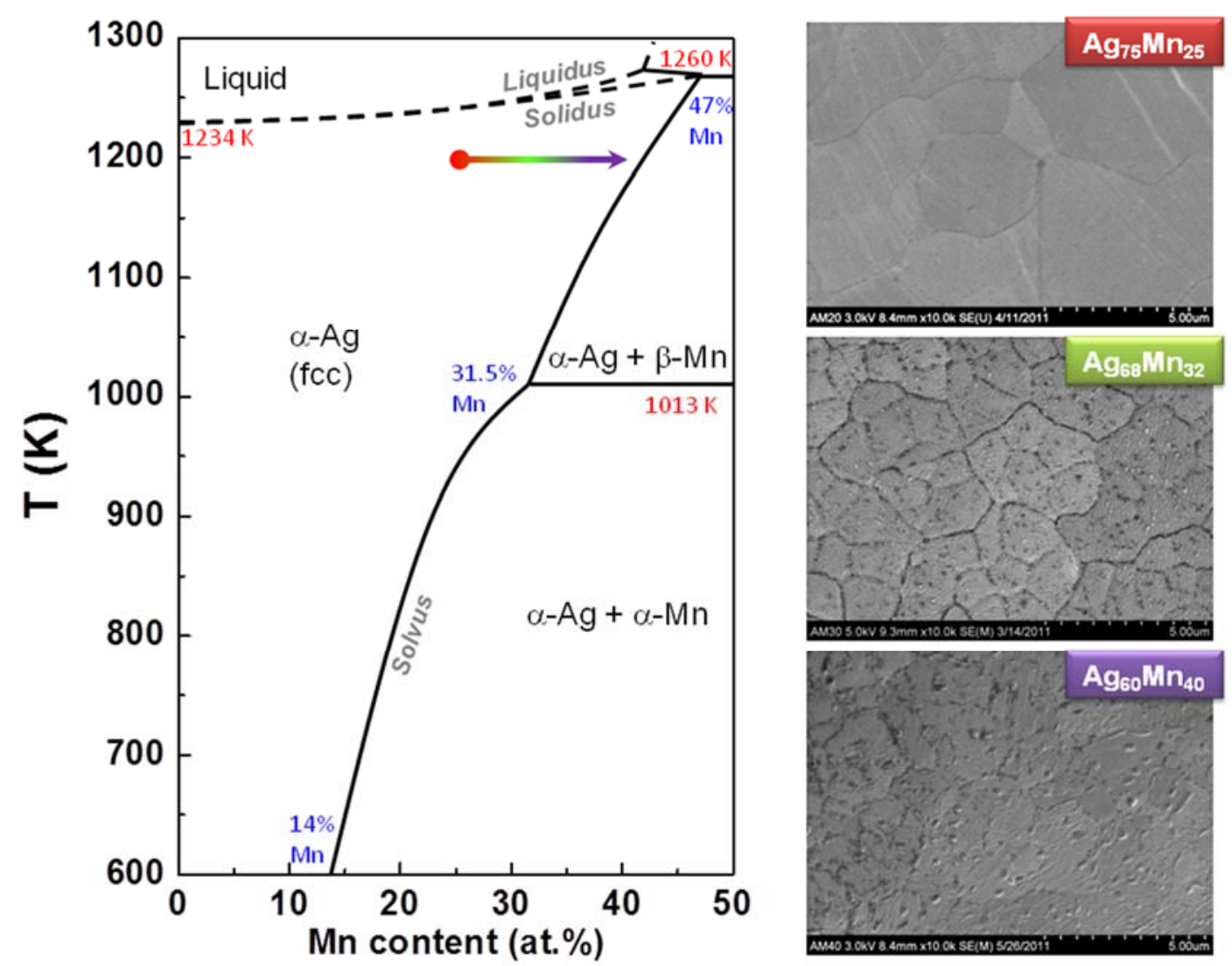

Fig. 2. Schema of phase diagram for Ag-Mn alloys at the Ag-rich region, based on Refs 20 and 56, highlighting (colored arrow) the quenching region for rapid solidification of $\mathrm{Ag}-\mathrm{Mn}$ alloys with $\mathrm{Mn}$ content between $[25,40]$ at.\%, and representative SEM images of the resulting quenched alloys. 
$\mathrm{X}$-ray diffractograms of the three samples $\left(\mathrm{Ag}_{100-\mathrm{x}} \mathrm{Mn}_{\mathrm{x}}, x=25,32,40\right)$ in their as-quenched state confirm a predominant face-centered-cubic $(f c c) \alpha$-AgMn phase constitution. Residual contributions of the $\alpha$-Mn phase to the XRD data appear with increased Mn content. A complex double Bragg-peak structure (see Figs. 3a-b) is observed for all measured peaks. The noted doubling of the major (111) Bragg reflection precludes symmetry lowering as the origin of the double-peak structure. Therefore it is deduced that the as-quenched ribbons consist of two $f c c$ phases of slightly different lattice parameter (denoted from here onwards as $\alpha_{1}$ and $\alpha_{2}$ for the $f c c$ phases with a large and a small unit cell volume, respectively). As expected, the lattice parameter decreases with increased Mn content for both phases (from $4.076 \AA$ to $4.059 \AA$ for $\alpha_{1}$, and from $a=$ $4.058 \AA$ to $a=4.050 \AA$ for $\alpha_{2}$, see Fig. 3c). The coherently-diffracting grain sizes, $L_{C}$, are estimated from the Williamson-Hall relationship as a function of increased Mn content; on average the crystallite sizes of the $\alpha_{1}$ phase, of larger unit cell volume, are smaller than those of $\alpha_{2}$ phase of smaller unit cell volume. It is interesting to note that although the lattice constant decreases with increased Mn content for both phases, the $\alpha_{l}$ phase crystallite size decreases to $L_{C} \sim 45 \mathrm{~nm}$ while the $\alpha_{2}$ phase crystallite size increases to $L_{C} \sim 85 \mathrm{~nm}$ (Fig. 3d). A clear increase of the strain is observed in the $\mathrm{Ag}_{60} \mathrm{Mn}_{40}$ sample ( $\eta$ increases from $\sim 6-9 \cdot 10^{-5}$ up to $\eta \sim 1.4-1.8 \cdot 10^{-3}$ ), see Fig. 3e), with respect to samples of lower Mn concentration. 

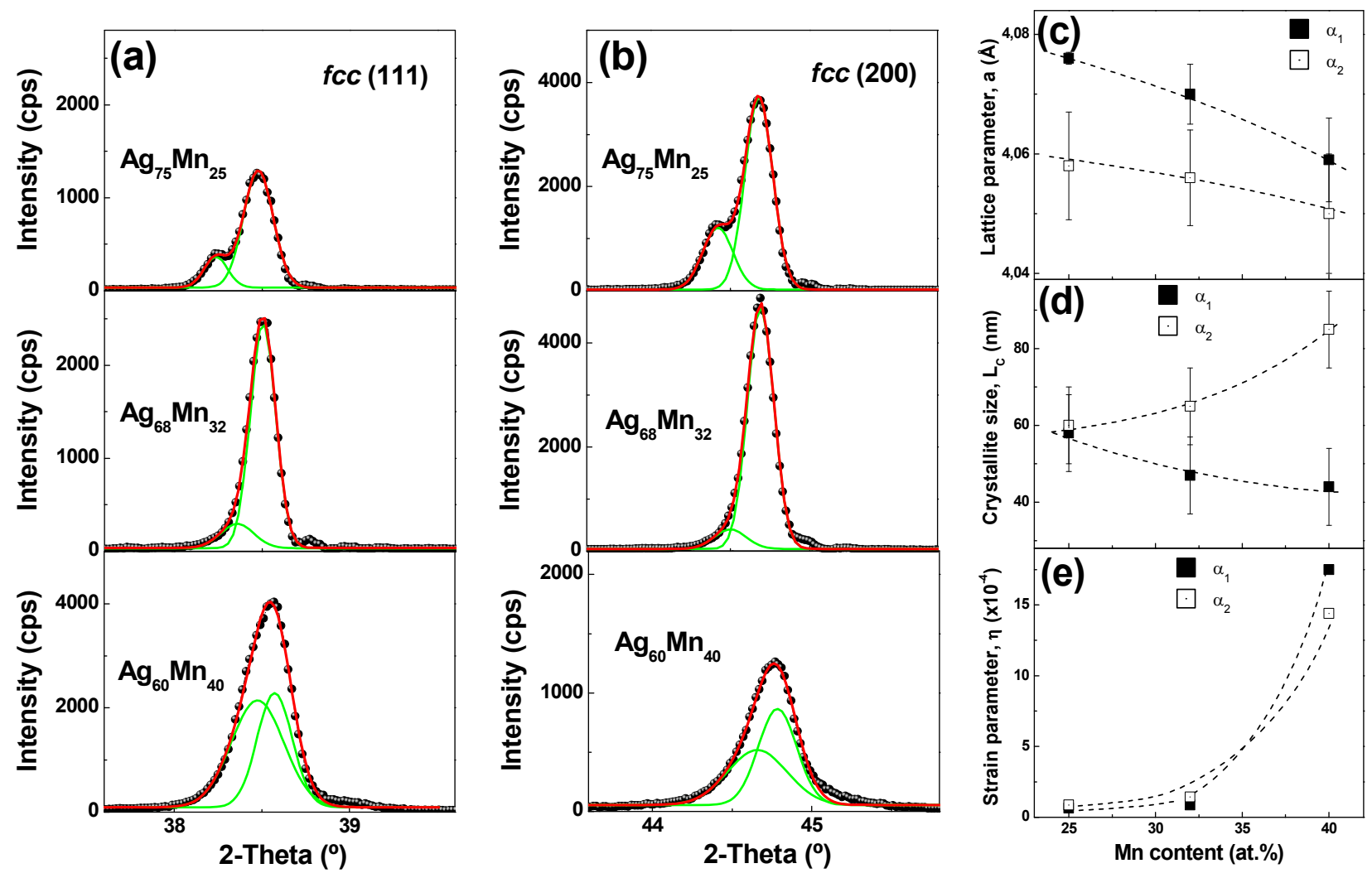

Fig. 3. (a) (111) and. (b) (200) x-ray diffraction peaks of the AgMn alloys, with a convenient two-peak fit. Evolution of the (c) lattice parameter (d) estimated average crystallite size, (e) strain, with the variation of the Mn content. Dash lines are included as a guide to the eye.

Neutron diffraction experiments were carried out on as-quenched $\mathrm{Ag}_{75} \mathrm{Mn}_{25}$ and $\mathrm{Ag}_{60} \mathrm{Mn}_{40}$ samples in a broad range of temperatures (diffractograms at $2 \mathrm{~K}$ are represented on Figs. $4 \mathrm{a}$ and $4 \mathrm{~b}$, respectively). Neutron and $\mathrm{x}$-ray diffraction patterns display the same Bragg features, and no additional Bragg peaks indicating long-order magnetic structure were detected. The absence of the double-peak pattern, noted in XRD, in any of the neutron diffraction experiments may be attributed to the lower resolution of the neutron diffractometer. A gradual decrease of the diffracted peak intensity with increased temperature is observed in all Bragg peaks and is accompanied by a shift to

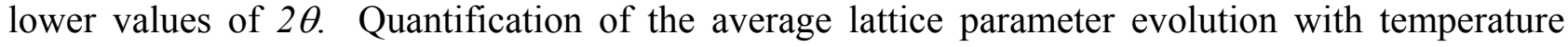
allows estimation of the thermal expansion coefficient of the system, calculated to be around $\varepsilon \approx$ 
$4 \times 10^{-3}$ at room temperature for both the $\mathrm{Ag}_{75} \mathrm{Mn}_{25}$ and $\mathrm{Ag}_{60} \mathrm{Mn}_{40}$ samples, Fig. 4d. This value is similar to values observed in other metallic Mn-based alloy systems. ${ }^{46}$
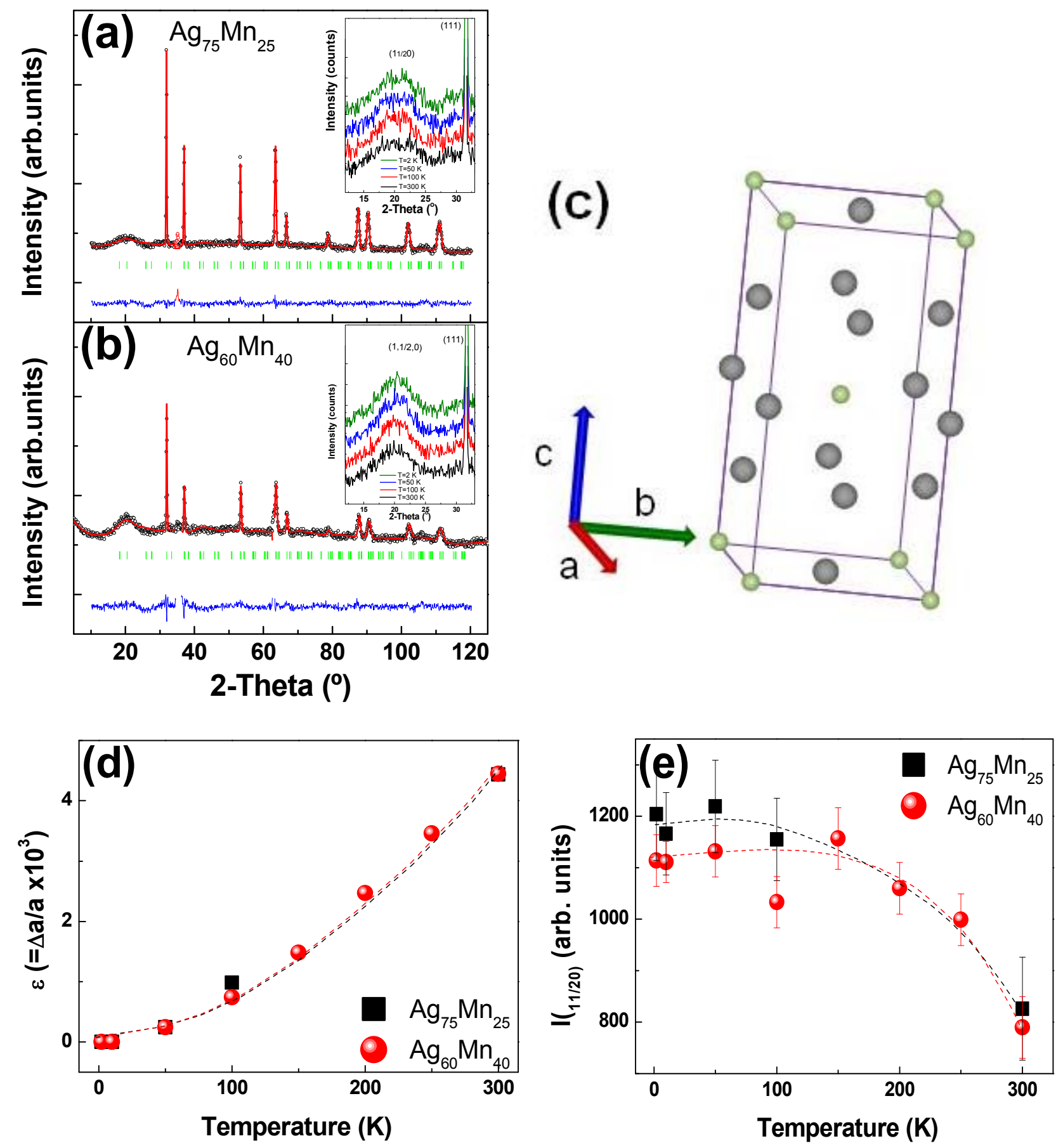

Fig. 4. Neutron powder diffraction patterns at $T=2 \mathrm{~K}$ (and best fits) for (a) $\mathrm{Ag}_{75} \mathrm{Mn}_{25}$ and (b) $\mathrm{Ag}_{60} \mathrm{Mn}_{40}$ alloys, and (c) proposed lattice model deduced from the fits (Insets: respective evolution of the diffuse peak with temperature, from 2 to $300 \mathrm{~K}$ ).. Evolution of (d) the thermal expansion coefficient, $\varepsilon$, and (e) the 
neutron diffuse $\left(1 \frac{1 / 20)}{2}\right.$ peak intensity with temperature, obtained from the neutron diffraction patterns of $\mathrm{Ag}_{75} \mathrm{Mn}_{25}$ and $\mathrm{Ag}_{60} \mathrm{Mn}_{40}$ alloys.

In addition, the neutron diffraction patterns exhibit a broad peak at around $2 \theta \approx 20^{\circ}$ that is associated with a diffuse scattering feature from reflections of the $\left(1 \frac{1 / 2}{2}\right)$ planes ${ }^{47,48}$. This feature is more intense and more narrow in the $\mathrm{Ag}_{60} \mathrm{Mn}_{40}$ sample than that displayed by the $\mathrm{Ag}_{75} \mathrm{Mn}_{25}$ sample. It is surmised that this diffuse scattering feature is too weak to be detected in laboratory-based x-ray diffraction experiments. A smooth decrease of the diffuse peak intensity with increased temperature (see insets of Figs. $4 \mathrm{a}$ and $4 \mathrm{~b}$, and Fig. 4e) is observed and persists to temperatures that are well above the magnetic blocking temperature previously reported for these Mn-based two-phase exchange-biased alloys ${ }^{22,49}$.

Rietveld refinement of the neutron data confirms an $f c c$ lattice (space group: $F m-3 m$; $\left.\chi^{2}=2.10 ; R_{B}=3.46\right)$ for the as-quenched alloys, with an estimated calculated stoichiometry of Mn atoms incorporated randomly within the Ag lattice that matches the results obtained by EDX analysis. ${ }^{17}$ The refinement also reveals the short-range nature of the diffuse $\left(1 \frac{1}{2} 0\right)$ peak. Refinement of the $\mathrm{Ag}_{75} \mathrm{Mn}_{25}$ and $\mathrm{Ag}_{60} \mathrm{Mn}_{40}$ diffraction patterns collected at $2 \mathrm{~K}$ reveals that the most suitable structural model to explain the origin of the diffuse scattering peak is a tetragonal cell, formed by two specular $f c c$ cells, where $c=2 a$, (space group: $P 4 / \mathrm{mmm} ; \chi^{2}=1.38 ; R_{B}=2.42$, as illustrated in Fig. 4c using the (001) direction as the tetragonal axis). This tetragonal superstructure can also be described as periodic juxtaposition of adjacent Mn (021) and Ag (021) planes (i.e., Mn (011/2) and Ag $\left(01 \frac{1}{2}\right)$ planes for the $f c c$ unit cell) ${ }^{50,51}$ From microstructural analysis of the neutron diffraction patterns, an apparent coherently diffracting size of $\sim 8 \AA$ is estimated, in full agreement with the size of two $f c c$ cells that are contributing to the short range order. 
Field-cooled hysteresis loops measured at $10 \mathrm{~K}$ from the as-quenched alloys exhibit a very large exchange bias shift (from $H_{E} \approx 2 \mathrm{kOe}$ for $\mathrm{Ag}_{75} \mathrm{Mn}_{25}$ up to $H_{E} \approx 17 \mathrm{kOe}$ for $\mathrm{Ag}_{60} \mathrm{Mn}_{40}$, Figs. 5a-c). The intrinsic coercivity $H_{C i}$ shows also very large values at low temperatures (from $H_{C i} \approx 5$ kOe for $\mathrm{Ag}_{75} \mathrm{Mn}_{25}$ up to $H_{C i} \approx 25 \mathrm{kOe}$ for $\left.\mathrm{Ag}_{60} \mathrm{Mn}_{40}\right)$. Both $H_{C i}$, and $H_{E}$ show a clear decrease in magnitude with increased temperature (see Figures $5 \mathrm{~d}-\mathrm{f}$ ) that becomes suppressed at temperatures close to the blocking temperature, $T_{B}$, as determined by magnetothermal curves (see fresh sample measurements, inset of Fig. 6a). $H_{E}$ disappears at $70 \mathrm{~K}$ for $\mathrm{Ag}_{75} \mathrm{Mn}_{25}$, whereas $H_{E}$ disappears at 100 $\mathrm{K}$ for $\mathrm{Ag}_{68} \mathrm{Mn}_{32}$ and $\mathrm{Ag}_{60} \mathrm{Mn}_{40}$ alloys. 

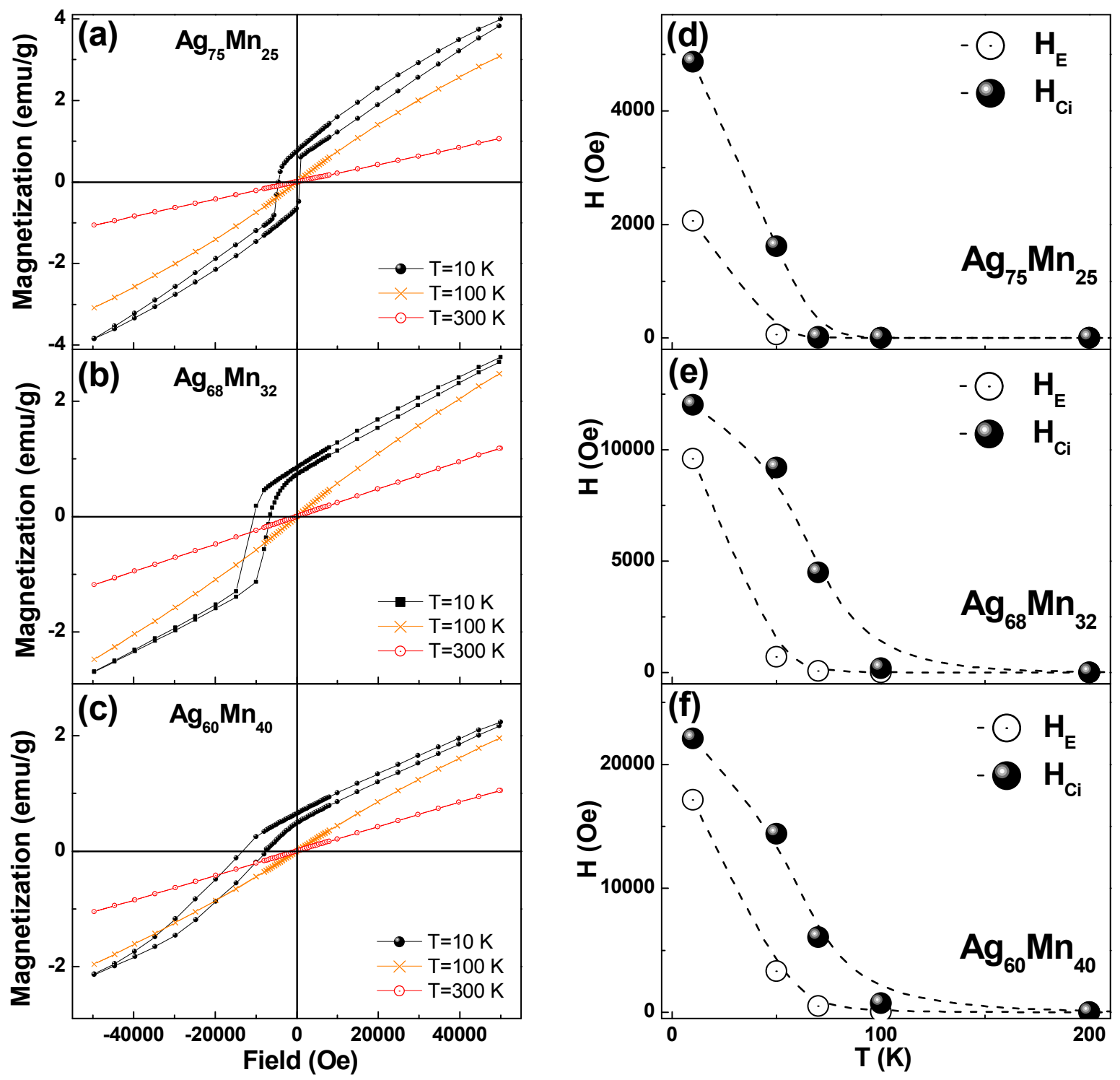

Fig. 5. Magnetization loops at different temperatures, for (a) $A_{9} M_{25}$, (b) $A_{6}{ }_{68} M_{32}$ and (c) $A_{60} M_{40}$;

Variation of $H_{E}$ and $H_{C i}$ with temperature for (d) $\mathrm{Ag}_{75} \mathrm{Mn}_{25}$, (e) $\mathrm{Ag}_{68} \mathrm{Mn}_{32}$ and (f) $\mathrm{Ag}_{60} \mathrm{Mn}_{40}$.

Annealing modifies the magnetic and structural features of the $\mathrm{Ag}_{68} \mathrm{Mn}_{32}$ alloy. After undergoing an annealing treatment of $350{ }^{\circ} \mathrm{C}$ the magnetization loops of the $\mathrm{Ag}_{68} \mathrm{Mn}_{32}$ sample measured at $T=10 \mathrm{~K}$ and at room temperature (Fig. 6a) as well as the magnetothermal ZFC-FC curves measured at $H=1 \mathrm{kOe}$ (inset) show an increased magnetization. Additional effects realized upon annealing are the suppression of the exchange bias and reduction of the blocking temperature peak, from $T_{B} \approx 105 \mathrm{~K} T_{B} \approx 60 \mathrm{~K}$. The x-ray diffraction pattern of the annealed $\mathrm{Ag}_{68} \mathrm{Mn}_{32}$ sample 
(Figure 6b) no longer evidences the double peak structure that was attributed to a two-phase system observed in the as-spun samples. Moreover, some residual (low intensity) peaks attributed to $\alpha$-Mn and $\mathrm{Mn}_{2} \mathrm{O}_{3}$ phases emerge after the heat treatment.
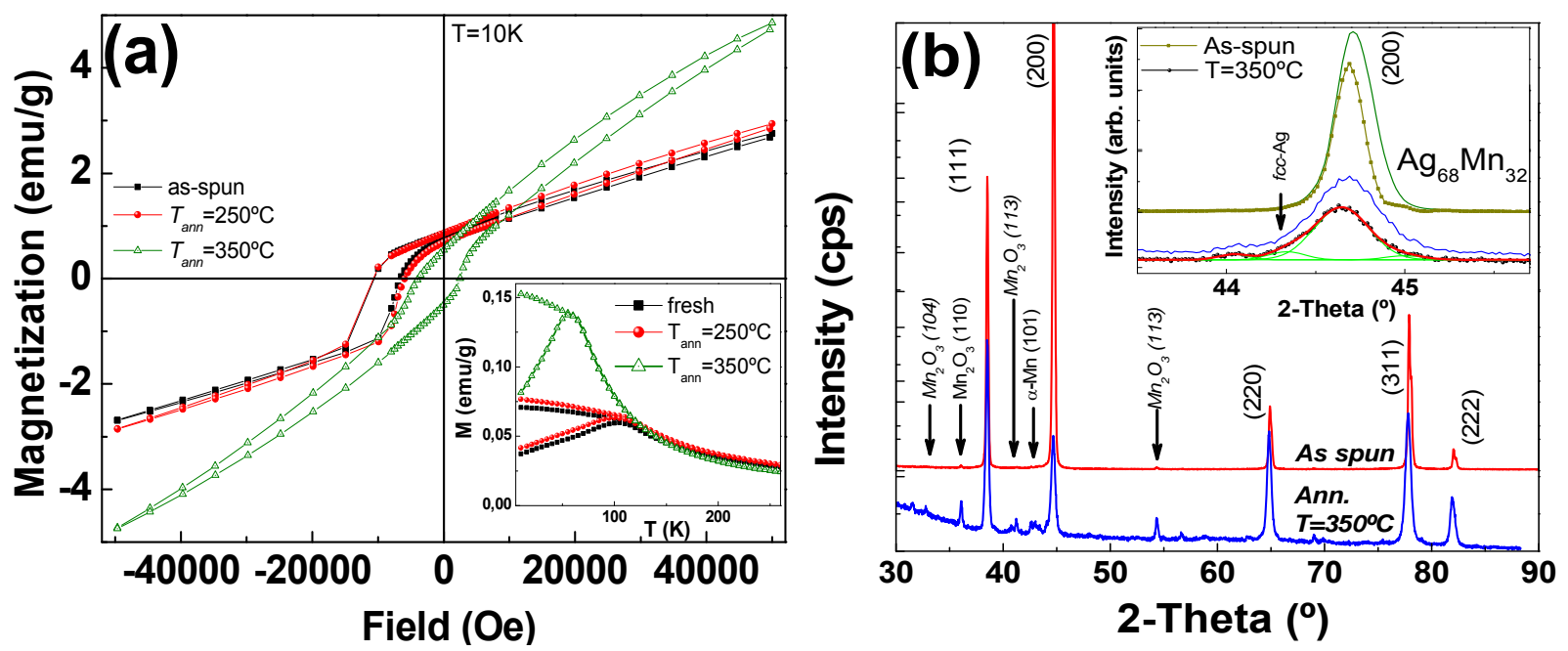

Fig.6. (a) Magnetization loops at $T=10 \mathrm{~K}$ of the as-spun and annealed $\mathrm{Ag}_{68} \mathrm{Mn}_{32}$ samples; (Inset: ZFC-FC curves at $H=1 \mathrm{kOe}$ of the fresh and annealed $\mathrm{Ag}_{68} \mathrm{Mn}_{32}$ sample); (b) X-ray diffraction patterns of the fresh and the annealed $\left(T_{a n n}=350{ }^{\circ} \mathrm{C}\right)$ ribbon; (Inset: fitting of the (200) peak).

\section{DISCUSSION}

Compilation of data obtained from the AgMn rapidly solidified materials confirms a heterogeneous compositional configuration at the nanoscopic scale that is attributed to uneven metastable entrapment of the solute upon solidification. Fluctuations in the Mn content produce phase segregation and a magnetic state with FM or AF behavior, lending appreciable exchange anisotropy at low temperatures. The concept is reminiscent of magnetic cluster glass described by classical spin-glass theory. ${ }^{52,53,54}$ Following the interpretation of microscopic exchange anisotropy model stated by Kouvel, ${ }^{35,55}$ random incorporation of Mn atoms form Mn-rich regions of strong AF 
character, due to enhanced contribution of antiparallel alignment of nearest-neighbor Mn magnetic moments, whereas Mn-poor areas demonstrate a finite magnetization attributed to next-nearest $\mathrm{Mn}$ neighbors with parallel spin alignment. The observed reduction in the AgMn lattice parameters with increased $\mathrm{Mn}$ content reflects incorporation of $\mathrm{Mn}$ into the $f c c$ lattice, with the $\alpha_{1}$ phase described as a Mn-poor phase whereas the $\alpha_{2}$ phase is a Mn-rich phase. Results confirm the intuitive increase of the nanocrystalline grain size of the Mn-rich phase as the Mn content is increased. The approximate $\mathrm{Mn}$ contents for each phase are determined assuming a linear relationship between the Mn content and the lattice parameters, employing the nominal literature values for fcc-Ag and for AgMn alloys with low Mn concentrations ${ }^{20,56}$, Figure 7a. In this manner the Mn concentration of the $\alpha_{2}(\mathrm{Mn}$-rich) phase in all three alloys is determined to be similar to that of the starting alloy (i.e., $x=25,32$ and 40 at.\% Mn). However, the Mn-deficient phases are determined to possess lower Mn concentrations, allowing the deduction that excess $\mathrm{Mn}$ was ejected from the lattice during solidification, forming Mn droplets that appeared on the alloy surface (see SEM images, Fig. 2) to give rise to nanoscale AgMn phases of ferromagnetic character.

These results are consistent with an enhanced concentration of first-neighbor Mn-Mn pairs in the as-solidified alloys, amplifying their antiferromagnetic character. However, no long-range AF (or FM) order was detected in these materials, confirming the dominance of short-range magnetic interactions. In mean-field-based theories, disorder is described by a random distribution of magnetic interactions between atoms. The fact that the diffuse peak is present throughout the whole range of temperatures, even above $T_{B}$, suggests a predominant nuclear nature of this diffraction feature, in agreement with previous literature ${ }^{47,57}$. In this sense, atomic short-range order modulates the magnetic short range arrangement ${ }^{58,59}$, yielding the aforementioned nanoscopic ferromagnetic and antiferromagnetic clusters according to their Mn concentration. Thus, a dual and interconnected 
atomic-magnetic nature of the short range order is present in these highly Mn-concentrated Ag-Mn alloys.
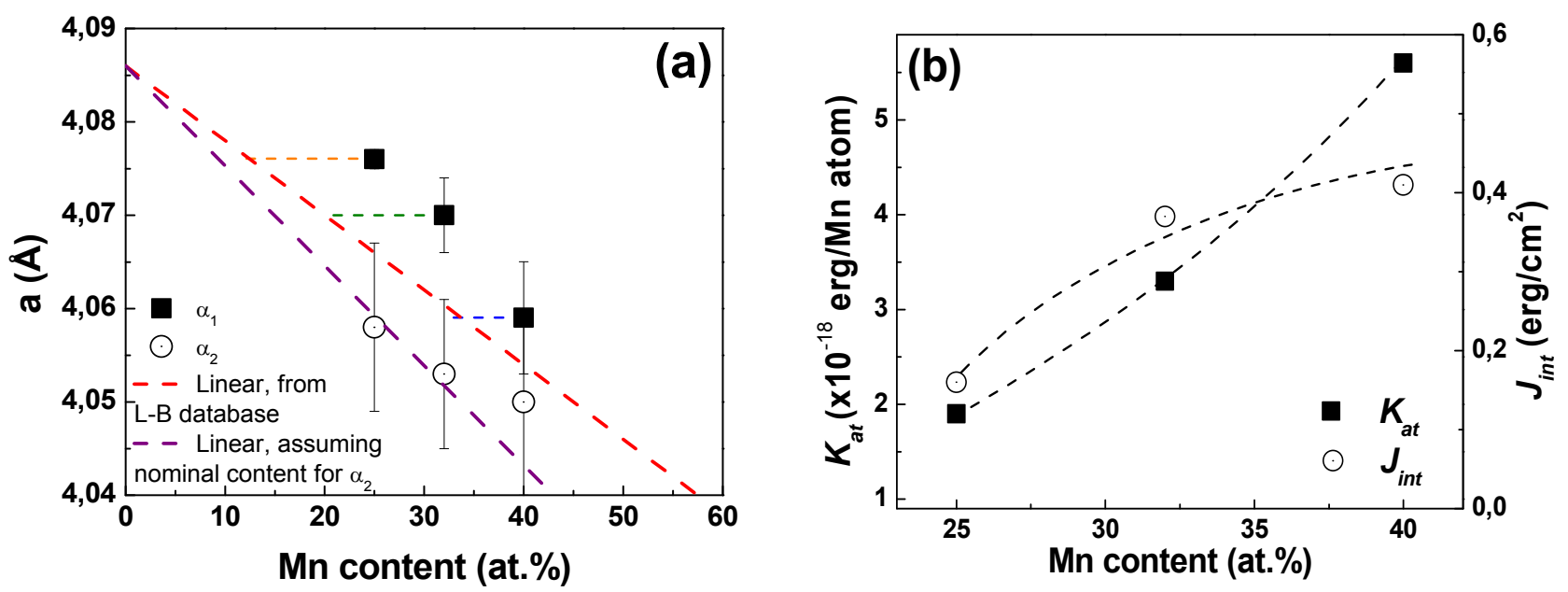

Fig. 7. (a) Estimation of Mn content using a linear relationship with the lattice parameter; (b) estimation of the unidirectional anisotropy coefficient, $K_{V}$ or $K_{a t}$, constant and the interface exchange energy, $J_{i n t}$, at $T=10$ $\mathrm{K}$, as a function of the Mn content.

The confirmation of nanocrystalline AgMn phases of differing Mn content and different magnetic character provides insight into the origins of the observed exchange bias. Average values of the interfacial exchange energy density $J_{\text {int }}$ between the Mn-deficient and Mn-rich regions may be estimated in these systems, assuming coherent rotation during demagnetization that does not result in domain formation ${ }^{37,60}$, as:

$$
J_{\text {int }} \approx H_{E} \cdot M_{F M} \cdot t_{F M}
$$

where $M_{F M}$ is the ferromagnetic phase magnetization $\left(M_{F M} \approx 1-2 \mathrm{emu} / \mathrm{g} \approx 1-2 \mathrm{Am}^{2} / \mathrm{kg}\right.$ in SI), obtained from the magnetometry measurements, and $t_{F M}$ is the average size of the coherentlydiffracted phase $\alpha_{1}\left(t_{F M} \equiv L_{C} \approx 44-58 \mathrm{~nm}\right)$ obtained from the XRD analysis. ${ }^{61}$ Utilizing magnetization results obtained at $T=10 \mathrm{~K}$, an increase of the interfacial exchange energy density is 
deduced with increased Mn content, from $J_{\text {int }} \approx 0.16 \mathrm{erg} / \mathrm{cm}^{2}\left(J_{\text {int }} \approx 1.6 \times 10^{-4} \mathrm{~J} / \mathrm{m}^{2}\right.$ in SI $)$ in $\operatorname{Ag}_{75} \mathrm{Mn}_{25}$ to $J_{\text {int }} \approx 0.46 \mathrm{erg} / \mathrm{cm}^{2}\left(J_{\text {int }} \approx 4.6 \times 10^{-4} \mathrm{~J} / \mathrm{m}^{2}\right.$ in $\left.\mathrm{SI}\right)$ for $\mathrm{Ag}_{60} \mathrm{Mn}_{40}$ (Fig. $\left.7 \mathrm{~b}\right)$. These values are comparable to those obtained from IrMn- or FeMn-based exchange-biased systems at room temperature $\left(J_{\text {int }} \approx 0.01-0.47 \mathrm{erg} / \mathrm{cm}^{2}\right) .{ }^{62}$ A significant increase in the unidirectional anisotropy coefficients at low temperatures with increased Mn concentration is observed, with magnitude provided per Mn atom following Kouvel's procedure ${ }^{35}$ (see Fig. 7b). Results of the unidirectional anisotropy range from $K_{a t} \approx 1.8 \times 10^{-18} \mathrm{erg} / \mathrm{Mn}$ atom for the sample with $x=25$ at.\% $\mathrm{Mn}$ to $K_{a t} \approx$ $5.6 \times 10^{-18} \mathrm{erg} / \mathrm{Mn}$ atom for $x=40$ at.\%. These values are enhanced over those reported by Kouvel for alloys with lower Mn concentration $\left(K_{a t} \approx 1-2 \times 10^{-18} \mathrm{erg} / \mathrm{Mn}\right)$. Overall, the combined structural, compositional and magnetic data are consistent with an increase in AF character and anisotropy strength with increased Mn content in the AgMn alloys. This quality exchange-hardens the ferromagnetic phase to prevent reorientation and reversal at low fields.

Data obtained from AgMn quenched samples subjected to annealing allows clarification of the relationships between the complex microstructure of the alloys and the existence of the noted exchange anisotropy effects. Annealing promotes disappearance of the double Bragg peak feature accompanied by a simultaneous shift in the Bragg peak positions; these effects are accompanied by suppression of the exchange bias in the low-temperature magnetization curves. These observations are consistent with homogenization of the two-phase microstructure and ejection of Mn through diffusion and subsequent aggregation. In fact, the presence of detectable $\alpha$ - $\mathrm{Mn}$ and $\mathrm{Mn}_{2} \mathrm{O}_{3}$ peaks in the XRD data reinforces the idea of induced manganese clustering via gradual Mn diffusion through the $f c c$ lattice. Annealing thus eliminates the magnetic differences between the original nanoscaled magnetic phases and serves as proof that exchange bias features in samples that exhibit this double peak diffracting structure as a signature of disparate Mn content. 


\section{CONCLUSIONS}

Rapidly-solidified $\operatorname{Ag}_{100-\mathrm{x}} \mathrm{Mn}_{\mathrm{x}}$ ribbons with a high metastable retention of $\mathrm{Mn}(25 \leq x \leq 40$ at.\%) were fabricated that display an extraordinary exchange bias at low temperature that increases with increased Mn content, up to $H_{E} \approx 17 \mathrm{kOe}$ at $10 \mathrm{~K}$, informing potential applications in tunable exchange-biased systems. Detailed microstructural characterization of the as-quenched materials revealed the existence of nanoscopic phase separation comprised of two majority $f_{c c}$ phases with slightly different lattice parameters, attributed to different concentrations of $\mathrm{Mn}$, resulting in either antiferromagnetic or ferromagnetic character. Neutron diffraction data confirm that short-range structural ordering dominates the magnetic character, with an average short-range structure size of $\sim 2 a$. The fact that annealing procedures convey diffusion of $\mathrm{Mn}$ and erases the phase-separated structure with simultaneous suppression of magnetization loop displacement at low temperature supports the scenario by which exchange bias stems from strong coupling of nanometric phases with irregular Mn content. Estimation of the Mn content of each nanometric region and their intitmate interaction suggests that the Mn-rich phase maintains the nominal Mn concentration, and that local diffusion of $\mathrm{Mn}$ gives rise to the Mn-poor phase, with large values of exchange coupling at the interface.

\section{ACKNOWLEDGEMENTS}

This research has been funded by the Office of Naval Research (ONR) under Grant \# N00014-10-10553. IPO acknowledges Spanish MINECO for financial support (MAT2011-23455 and MAT2011-25991). We thank the ILL and CRG-D1B beamline for allocation of neutron beam time and support during the experiments. 


\section{REFERENCES}

[1] W. H. Meiklejohn and C. P. Bean, Phys. Rev. 102, 1413 (1956).

[2] R. Morales, A. C. Basaran, J. E. Villegas, D. Navas, N. Soriano, B. Mora, C. Redondo, X. Batlle and I. K. Schuller, Phys. Rev. Lett. 114, 097202 (2015).

[3] H. Wu, C. H. Wan, Z. H. Yuan, X. Zhang, J. Jiang, Q. T. Zhang, Z. C. Wen, and X. F. Han, Phys. Rev. B 92, 054404 (2015).

[4] W. Zhang and K. M. Krishnan, Mater. Sci. Eng. R 105, 1 (2015).

[5] L. H. Lewis, C. L. Harland, R. W. McCallum, M. J. Cramer and K. W. Dennis, J. Appl. Phys. 99, 08E908 (2006).

[6] J. Sort, S. Suriñach, J. S. Muñoz, M. D. Baró, J. Nogués, G. Chouteau, V. Skumryev and G. C. Hadjipanayis, Phys. Rev. B 65, 174420 (2002).

[7] M.A. Laguna-Marco, J. Sánchez-Marcos, N. Menéndez, J. Chaboy, E. Salas-Colera and C. Prieto, Mater. Des. 93, 388 (2016).

[8] W.H. Meiklejohn and C.P. Bean, Phys. Rev. 105, 904 (1957).

[9] J. Sort, J. Nogués, S. Suriñach, J.S. Muñoz, M.D. Baró, E. Chappel, F. Dupont and G. Chouteau, Appl. Phys. Lett. 79, 1142 (2001).

[10] P. Toson, A. Asali, W. Wallisch, G. Zickler and J. Fidler, IEEE Trans. Magn. 51, 7400104 (2015).

[11] S. Liébana-Viñas, U. Wiedwald, A. Elsukova, J. Perl, B. Zingsem, Anna S. Semisalova, Verónica Salgueiriño, M. Spasova, and M. Farle, Chem. Mater., 27, 4015 (2015).

[12] L. Lin, N. Thiyagarayah, H. W. Joo, J. Heo, K. A. Lee and S. Bae, Appl. Phys. Lett. 97, 242514 (2010).

[13] K. Elphick, G. Vallejo-Fernandez, T. J. Klemmer, J.-U. Thiele and K. O’Grady, Appl. Phys. Lett. 109, 052402 (2016).

[14] O. E. Peil, A. V. Ruban and B. Johansson, New J. Phys. 10, 083026 (2008).

[15] M. Mizrahi, A. F. Cabrera, S. J. Stewart and J. Desimoni. J. Appl. Phys. 115, 213904 (2014).

[16] F. T. Yuan, J. K. Lin, Y. D. Yao and S. F. Lee, Appl. Phys. Lett. 96, 162502 (2010).

[17] E. Kneller and R. Hawig, IEEE Trans. Magn. 27 , 3588 (1991).

[18] L. H. Lewis and F. Jiménez-Villacorta, Metall. Mater. Trans. A 44, Suppl., 2 (2013).

[19] F. Jiménez-Villacorta, I. McDonald, D. Heiman and L. H. Lewis, J. Appl. Phys. 115, 17A729 (2014).

[20] The Landolt-Bornstein database: New Series, Group IV, Vol. 5, Subvolume a to j, B.Predel, O. Madelung, (ed.), Springer-Verlag (1991) to (1998).

[21] X. Ma, L. Li, Z. Zhang, H. Wang, E. Wang and T. Qiu, Mater.Des. 83, 1 (2015).

[22] F. Jiménez-Villacorta, J.L. Marion, T. Sepehrifar and L.H. Lewis, J. Appl. Phys. 111, 07E141 (2012). 
[23] F. Jiménez-Villacorta, J.L. Marion, T. Sepehrifar, M. Daniil, M. Willard and L.H. Lewis, Appl. Phys. Lett. 100, 112408 (2012).

[24] X. D. Tang, W.H. Wang, W. Zhu, E. K. Liu, G. H. Wu, F. B. Meng, H. Y. Liu and H. Z. Luo, Appl. Phys. Lett. 97, 242513 (2010).

[25] J. Sharma and K. G. Suresh, Appl. Phys. Lett. 106, 072405 (2015).

[26] P. Gibbs, T. M. Harders and J. H. Smith, J. Phys. F: Met. Phys. 15, 213 (1985).

[27] A. Banerjee and A. K. Majumdar, Phys. Rev. B 46, 8958 (1992).

[28] T. Taniguchi, T. Yamazaki, K. Yamanaka, Y. Tabata and S. Kawarazaki, J. Magn. Magn. Mater. 310, 1526 (2007).

[29] R. V. Chamberlin, M. Hardiman, L. A. Turkevich and R. Orbach, Phys. Rev. B 25, 6720 (1982).

[30] Q. Yang, P. Holody, S.-F. Lee, L. L. Henry, R. Loloee, P. A. Schroeder, W. P. Pratt Jr. and J. Bass, Phys. Rev. Lett. 72, 3274 (1994).

[31] J. Ederth, A. Hoel, C. I. Johansson, L. B. Kiss, E. Olsson, C. G. Granqvist and P. Nordblad, J. Appl. Phys. 86, 6571 (1999).

[32] T. Sato and K. Komatsu, J. Phys. D: Appl. Phys. 43, 474003 (2010).

[33] D. P. Morris and I. Williams, Proc. Phys. Soc. 73, 422 (1959).

[34] J. S. Kouvel, J. Phys. Chem. Solids 21, 57 (1961).

[35] J. S. Kouvel, J. Appl. Phys. 31, 142S (1960).

[36] J.-L. Liu, H.-Y. Huang and J.-X. Xie, Mater. Des. 64, 427 (2014).

[37] M. Ali, P. Adie, C.H. Marrows, D. Greig, B. J. Hickey and R.L. Stamps, Nat. Mater. 6, 70 (2007).

[38] W. Kuch, L. I. Chelaru, F. Offi, J. Wang, M. Kotsugi and J. Kirschner, Nat. Mater. 5, 128 (2006).

[39] G. A. Novak and A. A. Colville, American Mineralogist 74, 488 (1989).

[40] H. Bi, S. Li, Y. Zhang and Y. Du, J. Magn. Magn. Mater. 277, 363 (2004).

[41] L. H. Lewis, A. R. Moodenbaugh, D. O. Welch and V. Panchanathan, J. Phys. D: Appl. Phys. 34, 744 (2001).

[42] J. Rodríguez Carvajal, Physica B 55, 192 (1993).

[43] L. H. Lewis and K. M. Bussmann, Rev. Sci. Instrum. 67, 3537 (1996).

[44] L. Xue, H. Liu, L. Dou, W. Yang, C. Chang, A. Inoue, X. Wang, R.-W. Li and B. Shen, Mater. Des. 56 $227-231$ (2014).

[45] T.-Y. Liu, P. Yang, L. Meng and F.-Y. Lu, J. Alloys Compd. 509, 8337 (2011).

[46] T. Yokoyama and K. Eguchi, Phys. Rev. Lett. 110, 075901 (2013).

[47] K. Ishibashi, Y. Tsunoda, N. Kunitomi and J.W. Cable, Solid State Commun. 56585 (1985).

[48] E. R. Vance, J. H. Smith and T. M. Sabine, J. Phys. C: Solid State Phys. 3, S34 (1970)

[49] F. Jiménez-Villacorta, D. Heiman, J. L. Marion and L. H. Lewis, IEEE Magn. Lett. 4, 1000204 (2013). 
[50] D. De Fontaine, Acta Metall. 23, 553 (1975).

[51] H. Bouchiat, E. Dartyge, P. Monod and M. Lambert, Phys. Rev. B 23, 1375 (1981).

[52] L. C. Barnsley, E. M. A. Gray and C. J. Webb, J. Phys.: Condens. Matter 25, 086003 (2013).

[53] E. P. Wohlfarth, Phys. Lett. A 70, 489 (1979).

[54] J. A. Mydosh, Spin Glasses: An Experimental Introduction, Ed. Taylor and Francis, London (1993).

[55] J. S. Kouvel and C. D. Graham Jr., J. Appl. Phys. 30, 312S (1959).

[56] I. Karakaya and W. T. Thompson, Bull. Alloy Phase Diagrams 11, 480 (1990).

[57] G. E. Bacon and N. Cowlam, J. Phys. C: Solid State Phys. 3, 675 (1970).

[58] J. Jankowska-Kisielinska, K. Swiderska and K. Mikke, Acta Physica Pol. 117, 582 (2010).

[59] P. Wells and J. H. Smith, J. Phys. F: Metal. Phys. 1, 763 (1971).

[60] P. Nieves, D. Kechrakos, and O. Chubykalo-Fesenko, Phys. Rev. B 93, 064432 (2016).

[61] A. E. Berkowitz and K. Takano, J. Magn. Magn. Mater. 200, 552 (1999).

[62] J. Nogués and I. K. Schuller, J. Magn. Magn. Mater. 192, 203 (1999). 
Graphical Abstract
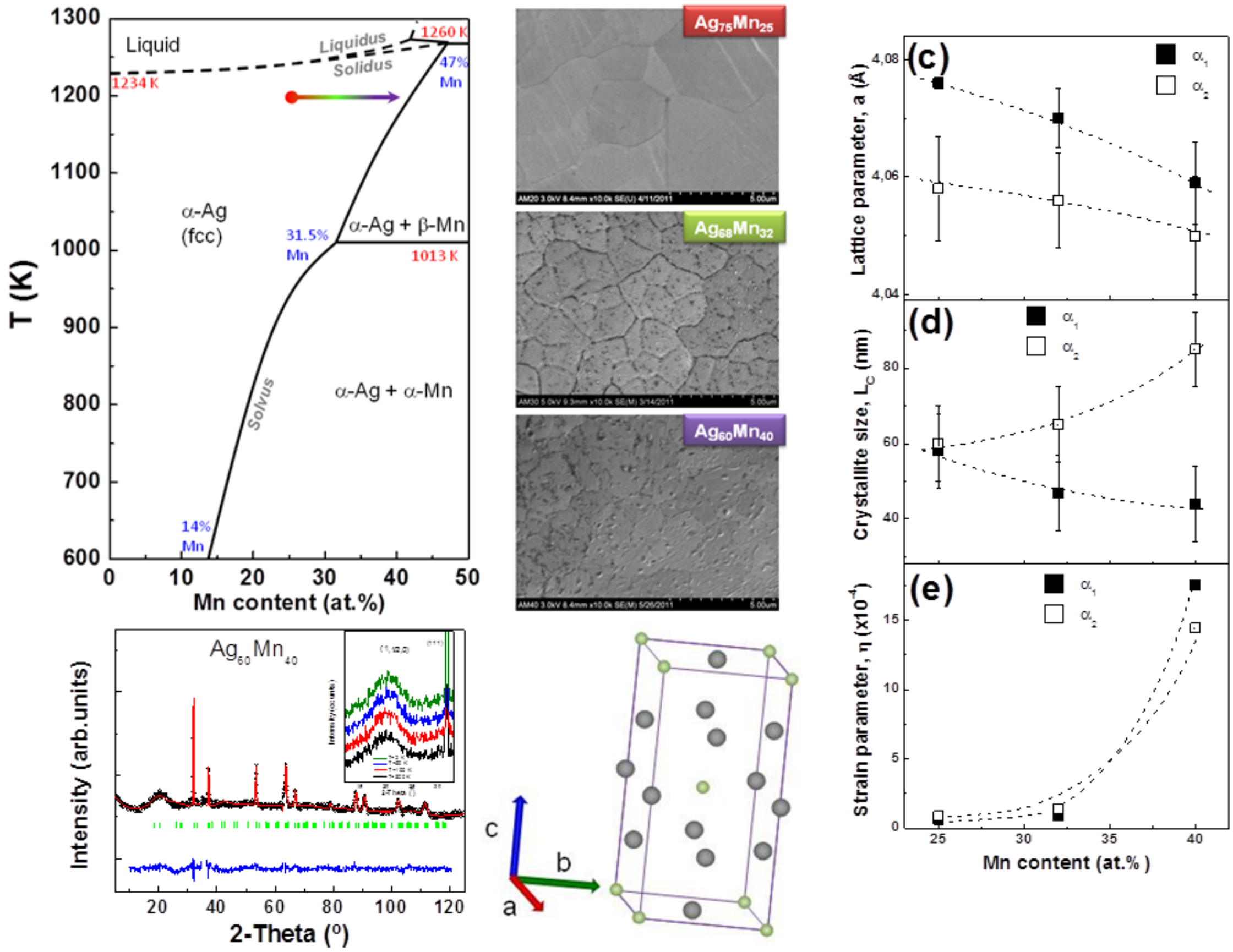